\title{
Biomechanical Analysis of the Proximal Femoral Locking Compression Plate: Do Quality of Reduction and Screw Orientation Influence Construct Stability?
}

\author{
Ivan Zderic, MSc, * Jong-Keon Oh, MD, †ł Karl Stoffel, MD, \& Christoph Sommer, MD,\| \\ Tobias Helfen, MD, 9 Gaston Camino, MD,** Geoff Richards, PhD, * Sean E. Nork, MD, †† \\ and Boyko Gueorguiev, PhD*
}

\begin{abstract}
Objectives: To investigate biomechanically in a human cadaveric model the failure modes of the proximal femoral locking compression plate and explore the underlying mechanism.
\end{abstract}

\begin{abstract}
Methods: Twenty-four fresh-frozen paired human cadaveric femora with simulated unstable intertrochanteric fractures (AO/OTA 31-A3.3) were assigned to 4 groups with 6 specimens each for plating with proximal femoral locking compression plate. The groups differed in the quality of fracture reduction and plating fashion of the first and second proximal screws as follows: (1) anatomic reduction with on-axis screw placement; (2) anatomic reduction with off-axis screw placement; (3) malreduction with on-axis screw placement; (4) malreduction with off-axis screw placement. The specimens were tested until failure using a protocol with combined axial and torsional loading. Mechanical failure was defined as abrupt change in machine load-displacement data. Clinical failure was defined as 5 degrees varus tilting of the femoral head as captured with optical motion tracking.
\end{abstract}

Results: Initial axial stiffness (in $\mathrm{N} / \mathrm{mm}$ ) in groups 1 to 4 was $213.6 \pm 65.0,209.5 \pm 134.0,128.3 \pm 16.6$, and $106.3 \pm 47.4$, respectively. Numbers of cycles to clinical and mechanical failure were $16,642 \pm 10,468$ and $8695 \pm 1462$ in group $1,14,076 \pm 3032$ and

Accepted for publication August 11, 2017.

From the *AO Research Institute Davos, Davos, Switzerland; $†$ Department of Orthopaedic Surgery, Guro Hospital, Korea University Medical Center, Seoul, Republic of Korea; \$Department of Orthopedic and Trauma Surgery, Cantonal Hospital Baselland, Liestal, Switzerland; †University of Basel, Basel, Switzerland; ||Department of General and Trauma Surgery, Cantonal Hospital Graubünden, Chur, Switzerland; PDepartment of General, Trauma and Reconstructive Surgery, Ludwig Maximilian University of Munich, Munich, Germany; **Hospital Italiano de Buenos Aires, Buenos Aires, Argentina; and ††Department of Orthopaedic Surgery, Harborview Medical Center, Seattle, WA.

This investigation was performed with the assistance of the AO Foundation via the AOTK System and supported by a grant from DePuy Synthes, Zuchwil, Switzerland.

The authors report no conflict of interest.

Presented in part at the Annual Meeting of the European Society of Biomechanics, July 4, 2017, Seville, Spain.

Supplemental digital content is available for this article. Direct URL citations appear in the printed text and are provided in the HTML and PDF versions of this article on the journal's Web site (www.jorthotrauma.com).

Reprints: Jong-Keon Oh, MD, Department of Orthopaedic Surgery, Guro Hospital, Korea University Medical Center, Seoul 08308, Republic of Korea (e-mail: jkoh@korea.ac.kr).

Copyright (C) 2017 Wolters Kluwer Health, Inc. All rights reserved.

DOI: 10.1097/BOT.0000000000001008
$7449 \pm 5663$ in group 2, $8800 \pm 8584$ and $4497 \pm 2336$ in group 3, and $9709 \pm 3894$ and $5279 \pm 4119$ in group 4 . Significantly higher stiffness and numbers of cycles to both clinical and mechanical failure were detected in group 1 in comparison with group $3, P \leq 0.044$.

Conclusions: Generally, malreduction led to significantly earlier construct failure. The observed failures were cut-out of the proximal screws in the femoral head, followed by either screw bending, screw loosening, or screw fracture. Proper placement of the proximal screws in anatomically reduced fractures led to significantly higher construct stability. Our data also indicate that once the screws are placed off-axis ( $>5$ degrees), the benefit of an anatomic reduction is lost.

Key Words: unstable intertrochanteric fracture, proximal femoral locking compression plate, reduction, screw placement, biomechanics

( Orthop Trauma 2018;32:67-74)

\section{INTRODUCTION}

Fractures in the trochanteric region are frequently caused by falls from standing height, with approximately $90 \%$ related to low-energy injuries and poor bone quality in elderly patients. ${ }^{1}$ Early operative treatment is usually required using either intramedullary or extramedullary implants. ${ }^{2}$ Intertrochanteric fractures are associated with postoperative mortality rates of up to $29 \%$, as reported in a 2-year follow-up study including patients treated with a sliding hip screw implant. ${ }^{3}$ Cephalomedullary nail insertion is considered to be the gold standard for unstable AO/OTA 31-A2 and A3 fractures. ${ }^{4,5}$

The proximal femoral locking compression plate (PF-LCP; DePuy Synthes, Zuchwil, Switzerland) was introduced as a fixation option in $2007 .{ }^{6}$ A high failure rate up to $41 \%$ has been reported after plating of intertrochanteric fractures with this implant. ${ }^{7-12}$ The most common failure mode was varus collapse frequently associated with screw cut-out. Proximal screw disengagement with fracture of the second proximal screw was identified as well. ${ }^{8}$ In their recent multicenter study, Collinge et $\mathrm{a}^{11}$ reported that in most cases proximal screws were the main source of PF-LCP failure.

Recently, Schneider et al biomechanically reproduced the failure mode observed in clinical cases with this implant by subtle (2 degrees) misplacement (off-axis placement) of the proximal locking screws and demonstrated that this small degree of screw malposition can lead to significantly earlier failure in 
contrast with on-axis screw placement. ${ }^{13}$ The authors suggested that subtle screw misplacement can occur in the operating theatre and thereby lead to catastrophic failure. Additionally, several clinical studies, analyzing the reasons for PF-LCP failures, almost unanimously stressed that it was crucial to avoid malreduction of the intertrochanteric fracture, especially in varus. ${ }^{7,11,14}$

The aim of this study was to assess the biomechanical effect of the most common malreduction, varus with apex anterior angulation, on the PF-LCP failure load and mode. Furthermore, it was sought to examine the influence of off-axis proximal screw placement on construct failure.

\section{MATERIALS AND METHODS}

\section{Specimens Preparation, Study Groups, and Fracture Model}

Twelve pairs of fresh-frozen $\left(-20^{\circ} \mathrm{C}\right)$ human cadaveric femora [ 6 female and 6 male donors, age $82 \pm 7$ years (mean \pm SD), range 71-96 years] were used in this study. Radiographs were taken to exclude previous pathologies and/or surgeries. Bone mineral density (BMD) was measured in the femoral head via high-resolution peripheral quantitative computed tomography using an Xtreme CT (Scanco Medical AG, Brüttisellen, Switzerland).

Based on BMD, the 24 specimens were randomized in 4 study groups for plating (1-4), consisting of 6 specimens each. Besides, groups 1 and 3, as well as groups 2 and 4 were paired, that is, containing paired femora. The 4 groups were with equal number of left and right bones and differed in the quality of fracture reduction and plating fashion as described below.

All specimens were thawed at room temperature 24 hours before preparation. Osteotomies were set to simulate unstable intertrochanteric fracture type AO/OTA 31-A3.3 with the following 3 components: (1) loss of posteromedial support by broken lesser trochanter; (2) broken lateral femoral wall; and (3) extension of the fracture line to the greater trochanter. ${ }^{15}$ The first osteotomy line was created between the tip of the greater trochanter and the medial cortex along the intertrochanteric line. The second osteotomy line was set perpendicular to the intertrochanteric line between the distal one-third of the former and the lateral cortex (Fig. 1A). The lesser trochanter was removed via a cut through its base. For an overview of the quality of fracture reduction and plating fashion in the 4 groups, see Supplemental Digital Content 1 (Table, http://links.lww.com/JOT/A169).

In group 1 (reduction/on-axis), the fragments were anatomically reduced and each femur was instrumented with a 4.5/5.0 PF-LCP plate (6 shaft holes; DePuy Synthes) according to the manufacturer's guidelines. ${ }^{6}$ Proximal fixation succeeded by using 3 cannulated locking screws $(5.0 \mathrm{~mm}$ and $7.3 \mathrm{~mm}$ ) in the following steps: (1) $2.5-\mathrm{mm}$ guide wire insertion; (2) drilling with a drill bit according to the manufacturer's surgical technique guide; and (3) screw insertion over the guide wire and locking it with $6 \mathrm{Nm}$ torque using a torque indicator. Each screw length was determined to achieve appropriate distance to subchondral bone. The distal 3 locking screws $(5.0 \mathrm{~mm})$ were inserted in shaft holes 2,5 , and 6 and locked with $6 \mathrm{Nm}$ torque.
In group 2 (reduction/off-axis), fracture reduction was anatomical; however, during plating, the first proximal screw was misplaced in 5 degrees posterior off-axis and the second screw in 5 degrees anterior off-axis. Off-axis orientation was realized by means of guide wire insertion through custom-made wire guides (Fig. 1B).

In group 3 (malreduction/on-axis), the fracture fragments were malreduced to simulate 5 degrees varus and 5 degrees apex anterior angulation. To achieve reproducible malreduction, a custom-made 5-degree wedge was first inserted in the intertrochanteric osteotomy line (Fig. 1C). The femoral head was then rotated posteriorly around its neck axis under controlled goniometer measurement (Fig. 1D). Plating was performed in the same fashion as for group 1.

In group 4 (malreduction/off-axis), fracture reduction was same as in group 3 and plating was same as in group 2 .

The amount of 5 degrees malreduction and off-axis screw placement were arbitrarily chosen considering diverse clinical situations with possible unsatisfactory anatomic fragment reductions, especially in minimally invasive approaches, also taking in consideration positioning and repositioning of the same guide wire that could lead to a small amount of unrecognizable screw malposition.

The distal part of each specimen was cut after plating $7 \mathrm{~cm}$ distally to the distal end of the plate and embedded in a polymethylmethacrylate (Suter Kunststoffe AG, Fraubrunnen, Switzerland) cylinder form. Subsequently, the femoral head was embedded in polymethylmethacrylate using a custom fixture (Fig. 1E). Retroreflective marker sets were attached to the femoral head fragment and the shaft for optical motion tracking.

\section{Biomechanical Testing}

Biomechanical testing was performed on a servo hydraulic testing machine (MTS 858 Bionix; MTS Systems Corp, Eden Prairie, MN). The setup for biomechanical testing is shown in Figure 2. Each specimen was mounted in 20 degrees adduction and 20 degrees flexion to simulate physiological peak forces acting on the femoral head during the mid-stance phase of the human gait ${ }^{16,17}$ and focus on proximal screw disengagement as a potential clinically relevant failure mode. $^{7-10}$ During the mid-stance phase the hip is flexed at 20 degrees while adducted at approximately 5 degrees. In addition, the direction of physiological hip contact force amounts to 12-16 degrees in the frontal plane, thus resulting in an angle of 20 degrees between the femur axis and the force direction, that is, in 20 degrees adduction of the femur specimen when tested with vertical force application. The proximal specimen embedding was fixed between 2 custom-made plastic plates connected via threaded steel rods allowing for fluoroscopic assessment. Vertical axial loading was applied to the specimen through a proximal cardan joint mounted to the machine actuator. The distal specimen embedding was fixed in a holder and attached to the machine base via a second cardan joint.

The loading protocol was adapted from a previous study $^{13}$ and comprised an initial nondestructive quasi-static axial tension ramp from 0 to $50 \mathrm{~N}$ at a rate of $25 \mathrm{~N} / \mathrm{s}$, followed by complex cyclic axial loading in compression-tension with a physiological profile of each cycle, applied at $2 \mathrm{~Hz}$ in combination with phased synchronic sinusoidal torsional loading in 
FIGURE 1. Photographic documentation of specimen preparation procedure. A, Two osteotomy lines simulating unstable intertrochanteric fracture AO/OTA 31-A3.3 with broken lateral femoral wall. B, Custommade wire guides attached to the first and second proximal plate holes for 5 degrees posterior and anterior off-axis guide wire insertion, respectively. C, A 5-degree wedge inserted in the intertrochanteric fracture line for varus malreduction. D, Goniometer used to measure 5 degrees rotation of the femoral head around the neck axis for apex anterior angulation. E, Custom-made fixture for femoral head embedding.

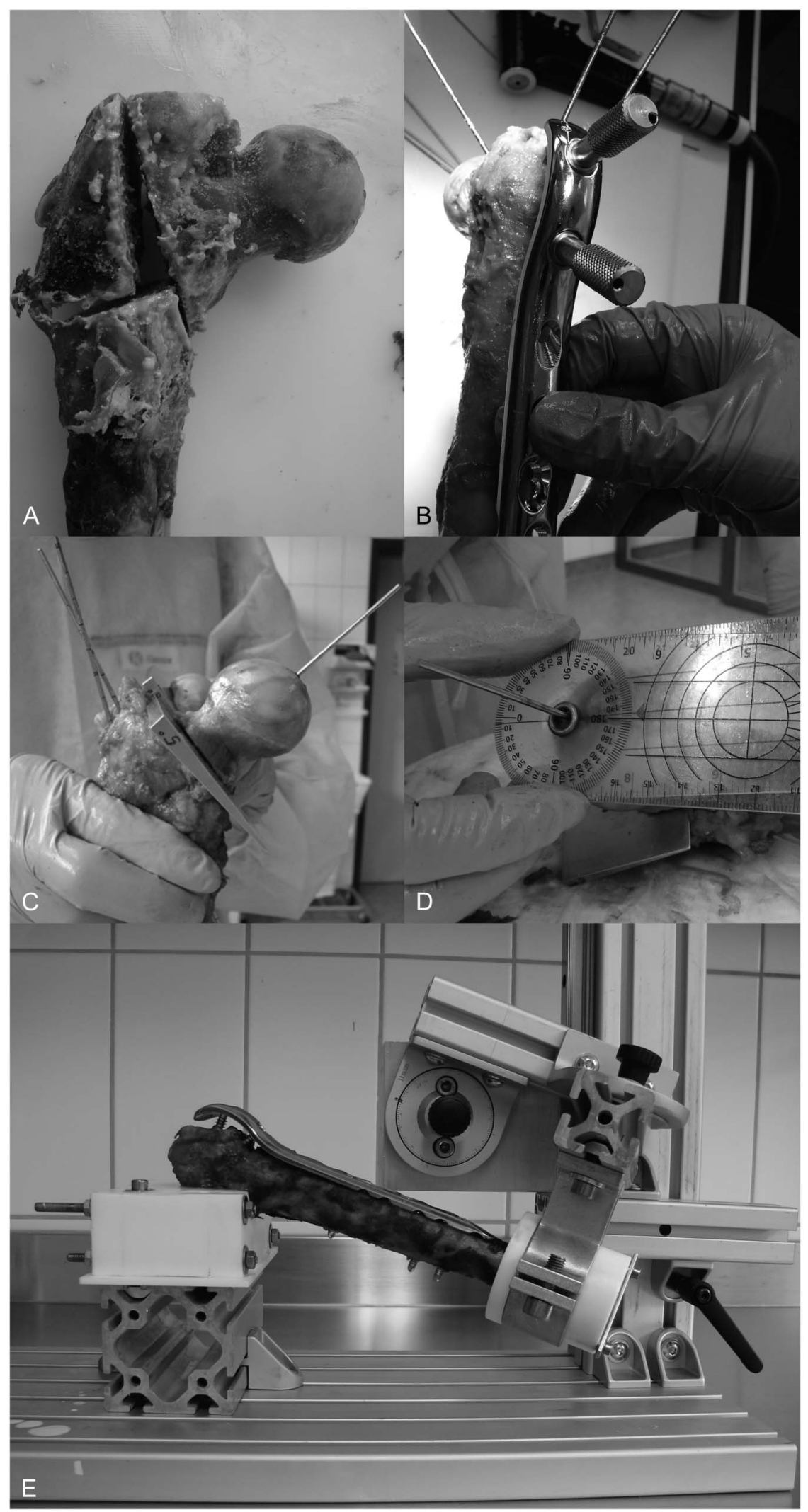


FIGURE 2. Setup for biomechanical testing. A, Anterior view of a right femur specimen mounted in 20 degrees adduction and 20 degrees flexion. Gray arrows denote axial and torsional loading direction. B, Posterior view of a left femur specimen with attached markers for optical motion tracking, ready for biomechanical testing.

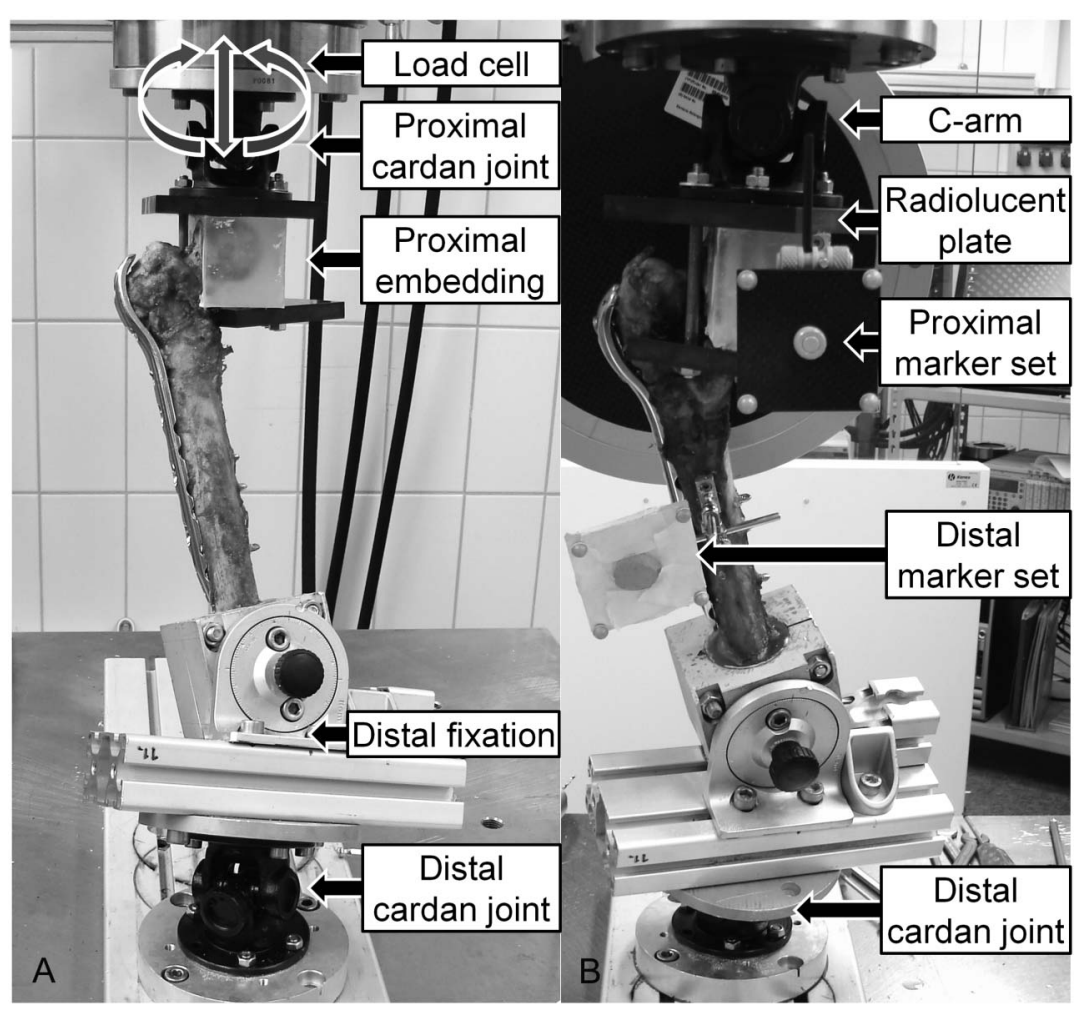

internal-external rotation. ${ }^{18}$ The peak torque in internal and external rotation was with constant amplitude of $6 \mathrm{Nm}$ around its average level of $0 \mathrm{Nm}$, kept in phase with the peak compression and tensile force, respectively (see Figure, Supplemental Digital Content 2, http://links.lww.com/JOT/A170, showing the cyclic loading over time). Whereas the peak tensile force was maintained constant at $50 \mathrm{~N}$, the peak compression increased cycle by cycle at a rate of $0.015 \mathrm{~N}$ per cycle, starting from $200 \mathrm{~N}$. The principle of cyclic testing with progressively increasing load has been found useful in previous studies. ${ }^{13,19,20}$ Alternating loading in compression-tension and internal-external rotation was reported to be crucial for crew disengagement. ${ }^{13}$ The tests were interrupted as soon as a distinct failure in the bone-plate construct was visually detected or when $50 \mathrm{~mm}$ actuator displacement or 30 degrees actuator rotation were reached.

\section{Data Acquisition and Analysis}

Machine data in terms of axial load $(\mathrm{N})$, displacement $(\mathrm{mm})$, torque $(\mathrm{Nm})$, and angle $\left(^{\circ}\right)$ was recorded from the test system's transducers at a sampling rate of $128 \mathrm{~Hz}$. Anteroposterior radiographic images were taken in the end of the quasi-static test and then at timed intervals every 500 cycles during the cyclic test at peak tensile load $(50 \mathrm{~N})$ using a triggered $\mathrm{C}$-arm. Interfragmentary movements of the femoral head with respect to the shaft were investigated by means of optical motion tracking at $100 \mathrm{~Hz}$ during the whole test with the use of 5 retroreflective cameras (Qualisys AB, Gothenburg, Sweden).

Parameters of interest were BMD, initial axial stiffness, translation after 8000 cycles, cycles to clinical and mechanical failure, load at clinical and mechanical failure, and failure

70 | www.jorthotrauma.com mode. Initial axial construct stiffness was calculated from the load-displacement machine data in the beginning of the cyclic test during the first loading cycle between 50 and 150 $\mathrm{N}$ compression. Translation was determined after 8000 test cycles as the magnitude of the 3-dimensional movement of the most superior osteotomy aspect, located on the femoral head fragment (see Figure, Supplemental Digital Content 3, http://links.lww.com/JOT/A171, showing the most superior osteotomy aspect of an instrumented specimen) under peak compression, with respect to its initial position in the beginning of the test and relative to the shaft. For that purpose, this point of interest was virtually separated in 2 aspects. The 2 aspects were then rigidly associated to either the shaft or the head markers, and their movements relative to each other were back-calculated by means of algorithms written in Matlab scripts (MathWorks, Natick, MA). Hence, it was assured that potential artifacts caused by shaft movements did not impair the results. The time point of evaluation represented the highest rounded cyclic number when none of the specimens had reached the test stop criterion yet. Cycles to clinical failure were defined as the number of cycles until reaching an arbitrary clinically relevant failure criterion of 5 degrees varus collapse of the femoral head. To consider only isolated fatigue-like behavior of the bone-implant constructs, the evaluation was performed under peak tension $(50 \mathrm{~N})$ with relatively low controllable loading of the specimens. During cyclic testing, the considerably bigger amount of applied compression in comparison with tension (higher than $200 \mathrm{~N}$ ) led to irreversible varus deformation of the femoral head, which was taken as baseline for evaluation. Cycles to mechanical failure were determined as the number of cycles 
until a rapid change in machine and radiological data, indicating either screw loosening or screw breakage, occurred. Load at clinical and mechanical failure was defined as the peak load of the corresponding cycle fulfilling the criterion for clinical or mechanical failure, respectively.

\section{Statistical Analysis}

Statistical analysis of the parameters of interest was performed using IBM SPSS Statistics software (v.22; IBM, Armonk, NY). Descriptive statistics was used to report all outcomes in terms of mean and SD values. Normal distribution of the data was screened with Shapiro-Wilk test. Significant differences between the groups consisting of paired specimens were investigated with paired samples $t$ test. General linear model repeated measures test was applied to explore the influence of BMD. Multiple comparisons among the groups with nonpaired specimens were performed by means of univariate analysis of variance with Bonferroni or Games-Howell post-hoc tests. Level of significance was set to 0.05 for all statistical tests.

\section{RESULTS}

\section{Bone Mineral Density}

BMD was not significantly different between the 4 groups (group 1: reduction/on-axis, $215.9 \pm 57.5 \mathrm{mg} \mathrm{HA} / \mathrm{cm}^{3}$; group 2: reduction/off-axis, $232.4 \pm 73.9 \mathrm{mg} \mathrm{HA} / \mathrm{cm}^{3}$; group 3: malreduction/on-axis, $213.9 \pm 48.0 \mathrm{mg} \mathrm{HA} / \mathrm{cm}^{3}$; group 4: malreduction/off-axis, $\left.216.2 \pm 70.0 \mathrm{mg} \mathrm{HA} / \mathrm{cm}^{3} ; P=0.959\right)$.

\section{Initial Axial Stiffness}

Initial axial stiffness was $213.6 \pm 65.0 \mathrm{~N} / \mathrm{mm}$ in group 1, $209.5 \pm 134.0 \mathrm{~N} / \mathrm{mm}$ in group $2,128.3 \pm 16.6 \mathrm{~N} / \mathrm{mm}$ in group 3 , and $106.3 \pm 47.4 \mathrm{~N} / \mathrm{mm}$ in group 4 (see Figure, Supplemental Digital Content 4, http://links.lww.com/JOT/A172, showing initial axial stiffness in the 4 groups). Group 1 revealed significantly higher stiffness in comparison with group $3, P=0.021$, with no further significances between the groups, $P \geq 0.196$. BMD had no significant influence on axial stiffness, $P=0.139$.

\section{Translation}

Translation after 8000 cycles was $11.6 \pm 8.8 \mathrm{~mm}$ in group $1,13.3 \pm 12.4 \mathrm{~mm}$ in group $2,16.8 \pm 6.7 \mathrm{~mm}$ in group 3 , and $17.7 \pm 4.2 \mathrm{~mm}$ in group 4 , with no significant differences between the groups, $P \geq 0.334$ (Fig. 3). BMD did not influence significantly the translation after 8000 cycles, $P=0.115$.

\section{Cycles and Load to Clinical and Mechanical Failure}

Number of cycles to clinical failure was 16,642 $\pm 10,468$ in group $1,14,076 \pm 3032$ in group $2,8800 \pm 8584$ in group 3 , and $9709 \pm 3894$ in group 4 (Fig. 4). The corresponding failure load was $449.6 \pm 157.0 \mathrm{~N}$ in group $1,411.1 \pm 45.5 \mathrm{~N}$ in group 2, 332.0 $\pm 128.8 \mathrm{~N}$ in group 3 , and $345.6 \pm 58.4 \mathrm{~N}$ in group 4. Group 1 revealed significantly higher values than group $3, P=0.044$. No further significances were detected

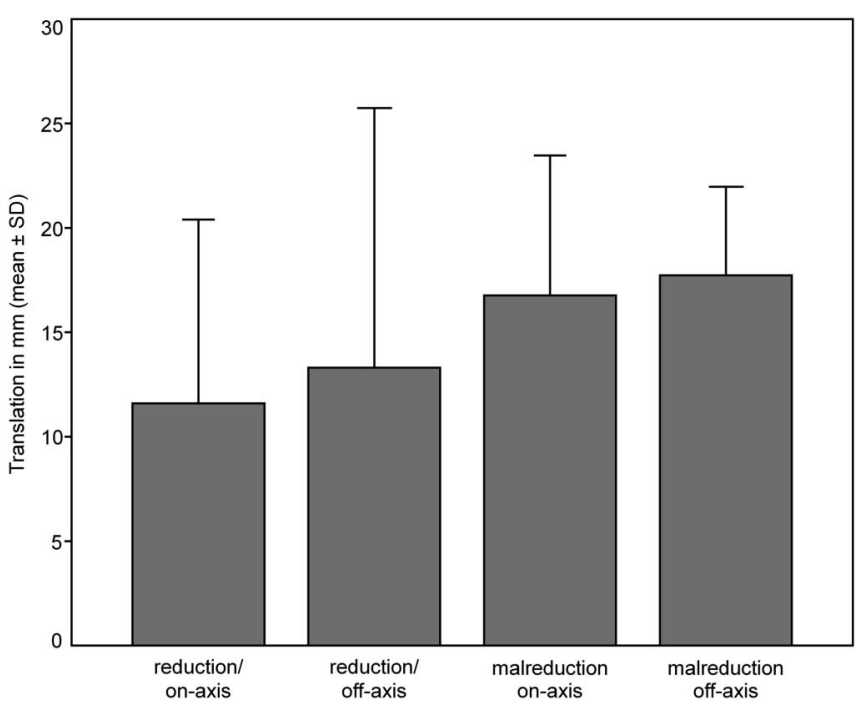

FIGURE 3. Diagram representing translation of the most superior osteotomy aspect after 8000 cycles in the 4 study groups.

among the 4 groups, $P \geq 0.111$. BMD influenced significantly cycles to clinical failure, $P=0.046$.

Number of cycles to mechanical failure was $8695 \pm$ 1462 in group $1,7449 \pm 5663$ in group 2, $4497 \pm 2336$ in group 3, and $5279 \pm 4119$ in group 4 (Fig. 4). The corresponding failure load was $330.4 \pm 21.9 \mathrm{~N}$ in group 1 , $311.7 \pm 84.9 \mathrm{~N}$ in group 2, $267.5 \pm 35.0 \mathrm{~N}$ in group 3 , and $279.2 \pm 61.8 \mathrm{~N}$ in group 4 . Group 1 was with significantly higher values compared with group $3, P=0.019$. No further significances were observed among the 4 groups, $P \geq 0.543$. BMD showed no significant influence on cycles to mechanical failure, $P=0.553$.

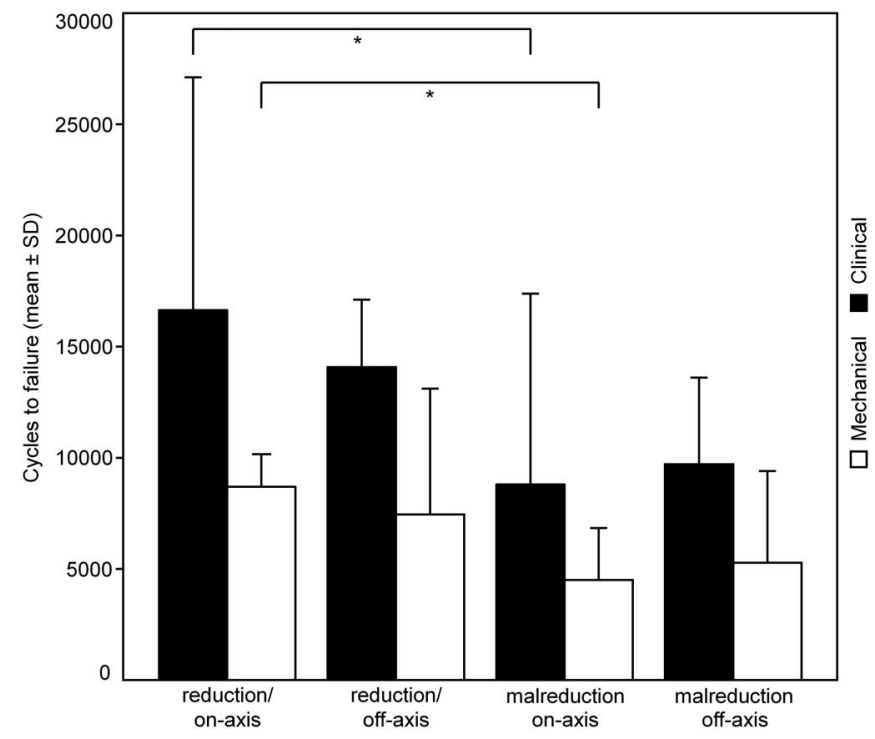

FIGURE 4. Diagram representing cycles to clinical and mechanical failure in the 4 study groups with stars indicating significant differences. 

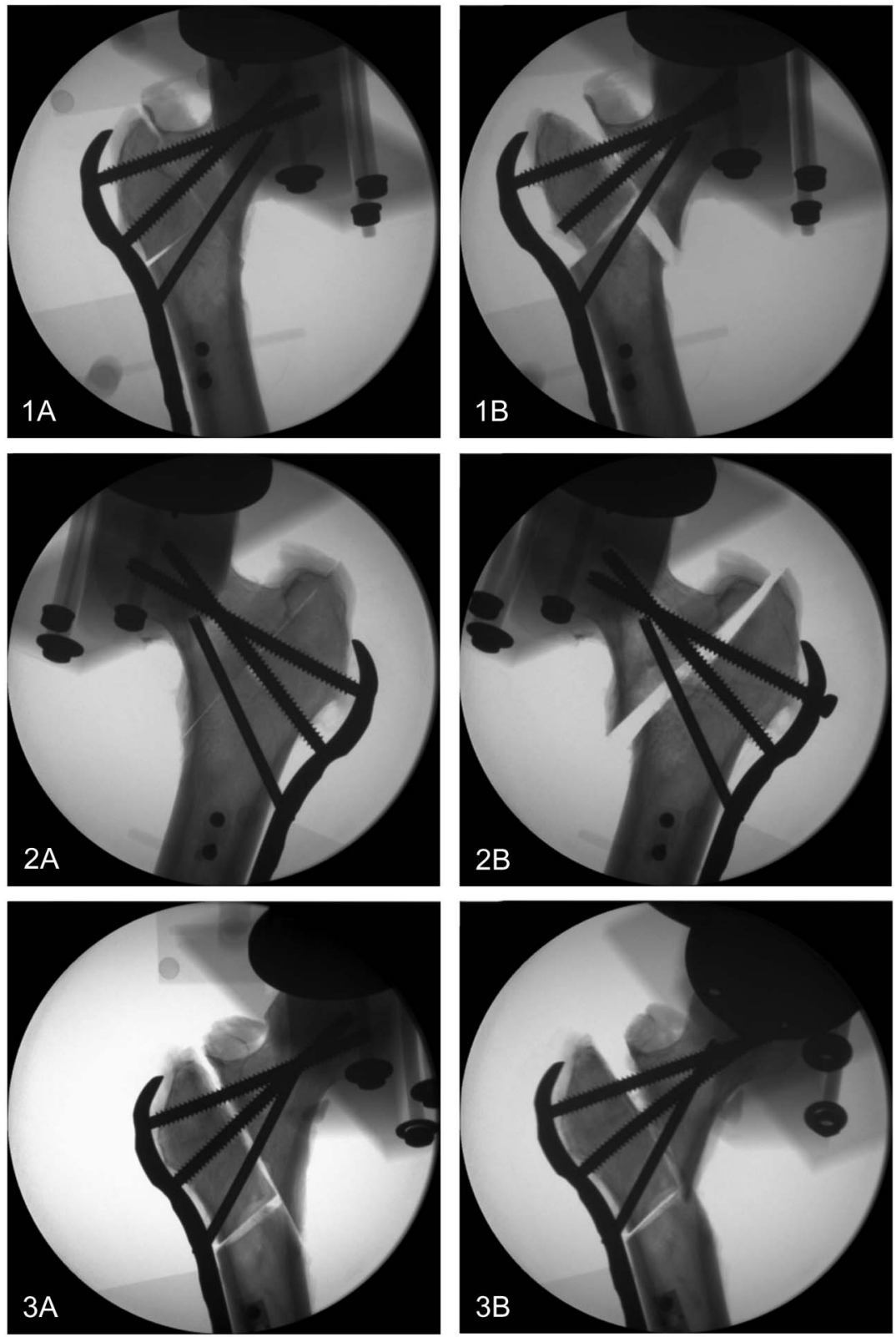

FIGURE 5. Anteroposterior radiographs of exemplified specimens in the 4 study groups (1: anatomic reduction/on-axis screw placement; 2: anatomical reduction/off-axis screw placement; 3: malreduction/on-axis screw placement; and 4: malreduction/off-axis screw placement) before testing $(1 \mathrm{~A}, 2 \mathrm{~A}, 3 \mathrm{~A}$, and $4 \mathrm{~A})$ and after testing (1B, 2B, 3B, and 4B). Radiograph $1 B$ indicates loss of reduction and breakage of the second proximal screw. Radiograph $2 \mathrm{~B}$ indicates loss of reduction and loosening of the first proximal screw. Radiograph 3B indicates varus collapse of the femoral head and bending of the second proximal screw. Radiograph 4B indicates varus collapse of the femoral head and loosening of the second proximal screw.
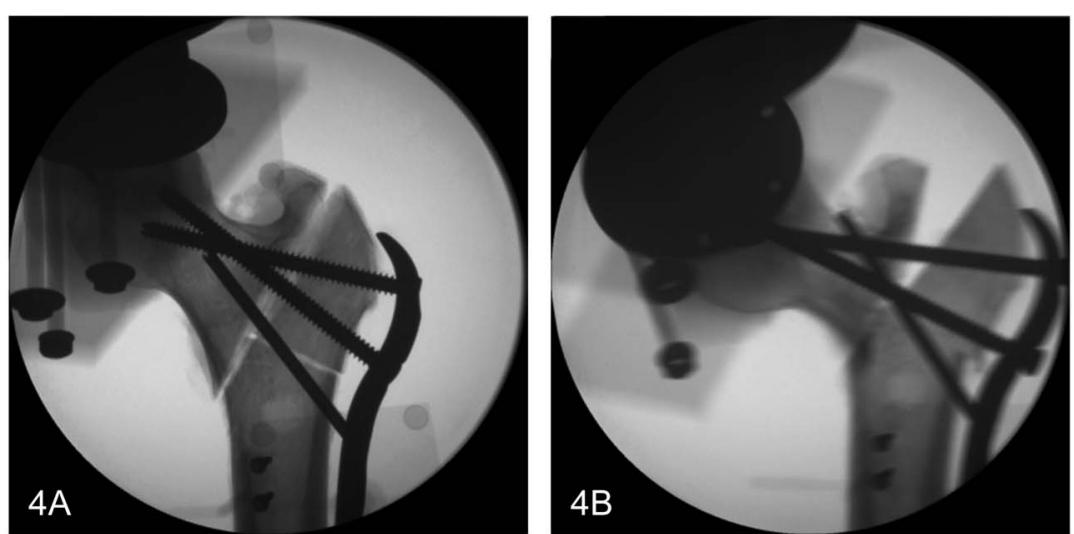
In terms of cycles, the mechanical failure criterion was fulfilled significantly earlier than the clinical one in all groups, $P=0.029$.

\section{Failure Mode}

Primary onset of failure in each specimen and group was exclusively cut-out of the proximal screws through the cancellous bone in the femoral head. In addition, screw loosening of the 2 proximal screws was observed in 4 specimens of group 4 (with a malreduced fracture and offaxis screw angulation). In group 2 (with a reduced fracture and off-axis screw angulation), the first proximal screw loosened in 2 femora. Bending of the first 2 proximal screws was observed in one pair of specimens assigned to groups 1 and 3 (with on-axis screw placement). Finally, fracture of the second proximal screw occurred in one specimen of group 1 . Representative radiographic images before testing and after specimen failure are shown in Figure 5.

\section{DISCUSSION}

The aim of this study was to biomechanically evaluate the impact of malreduction and off-axis screw insertion on stability of unstable intertrochanteric fractures fixed with a PF-LCP plate. Generally, malreduction led to significantly earlier construct failure. The observed failures were cut-out of the proximal screws in the femoral head, followed by either screw bending, screw loosening, or screw fracture.

Restoration of anatomy is the first principle of fracture management, and the rate of intertrochanteric malreduction can be as high as $30 \%{ }^{21}$ As with all implants used to secure an unstable intertrochanteric fracture, the quality of the reduction remains the most important factor for primary stability. When using the PF-LCP, however, this anatomic reduction must inevitably be combined with the placement of on-axis screws.

In the current study, the anatomically reduced specimens with properly inserted screws revealed significantly higher initial stiffness and cycles to failure as compared with nonanatomically reduced specimens. Importantly, our data also indicated that once the screws were placed off-axis, the benefit of the anatomic reduction was lost. The harmful effect of off-axis screw insertion has been demonstrated and discussed in previous biomechanical studies. ${ }^{13,22-25}$

The exclusive failure mode in the present study was cut-out of the 2 proximal screws through cancellous bone. This is reflected by the fact that mechanical failure, defined by abrupt change in the machine and radiological data, was reached significantly earlier than the clinical failure defined by 5 degrees varus tilting of the femoral head. Based on this, the mechanical failure can in fact be considered as primary clinical failure, whereas 5 degrees varus tilting would represent a secondary clinical failure, as a consequence of continued cyclic loading. Because the mechanical axis of the testing passed through the femoral head, BMD was found to significantly influence the results in regard to clinical failure.

This study has some limitations that are similar to those inherent to all cadaveric biomechanical investigations. First, a limited sample number was used, restricting a generalization to all patients. However, BMD was equally distributed among the groups assuring comparable conditions in this respect. Second, the groups were randomized in a way that a paired comparison between on-axis and off-axis screw placement was not possible. However, based on the fact that this phenomenon has previously been addressed, the main focus on investigation of the impact of fracture reduction seemed appropriate. Third, in contrast to some previous biomechanical studies, axial and torsional loading was applied with the femoral axis kept at constant inclination. Those studies investigated failure modes of different lag screw designs in intertrochanteric fracture models by introducing multiplanar loading setups to better simulate physiological loading environment during level walking or sit-to-stand activities and address clinically relevant failure modes. ${ }^{26-28}$ In their study, Ehmke et al used biaxial loading capability (axial and torsional) for application of the resultant loading vector upon the femoral head, ${ }^{26}$ reporting significant differences between multiplanar and uniplanar loading modes in terms of rotation around the implant axis. Building on these results, Born et al simulated flexion/extension at the hip by moving the femoral head in oscillating fashion under application of vertical load ${ }^{27}$ and demonstrating significantly increased predisposition to failure of their surrogate foam models in comparison with testing with constant specimen's inclination. In another study, Santoni et al adopted the oscillating test setup to compare InterTAN (Smith and Nephew, London, United Kingdom) versus Gamma 3 (Stryker, Kalamazoo, MI) lag screws in an inverted cadaveric hemi-pelvis model ${ }^{28}$ and were able to reproduce clinically relevant failures manifested by posterior head rotation, varus collapse, and cut-out.

The strengths of this study are based on the fact that all PF-LCP failure types, reported in previous investigations, such as plate fracture, loss of fixation (screw cut-out), screw bending, fracture, or loosening, were observed in the current testing environment with exception of the former, thus indicating the suitability of the applied test protocol with complex axial and torsional loading. ${ }^{8}$ Moreover, the varus collapse as frequently observed failure with the PF-LCP justifies the appropriate definition of the failure criteria used. ${ }^{7,8,10}$ Additionally, failure loads were in a physiological range and support the setup used. Finally, use of a reliable motion tracking system allowed for precise and meaningful interfragmentary motion analysis.

In conclusion, extrapolating from our biomechanical model, when using this device, on-axis placed proximal screws, combined with an anatomically reduced fracture is required to avoid mechanical failure. When using the PF-LCP, the benefit of an anatomic reduction is impeded by screw malposition as little as 5 degrees off-axis.

\section{ACKNOWLEDGMENTS}

The authors kindly thank Dieter Wahl and Benno Dicht for their support in setup development.

\section{REFERENCES}

1. Russell TA. Intertrochanteric fractures. In: Buchholz RW, Heckman JD, Court-Brown CM, et al, eds. Rockwood And Green's Fractures in 
Adults. 7th ed. Philadelphia, PA: Lippincott Williams \& Wilkins; 2010: 1597-1640.

2. Zha GC, Chen ZL, Qi XB, et al. Treatment of pertrochanteric fractures with a proximal femur locking compression plate. Injury. 2011;42: 1294-1299.

3. Ekstrom W, Miedel R, Ponzer S, et al. Quality of life after a stable trochanteric fracture-a prospective cohort study on 148 patients. J Orthop Trauma. 2009;23:39-44.

4. Barton TM, Gleeson R, Topliss C, et al. A comparison of the long gamma nail with the sliding hip screw for the treatment of AO/OTA 31-A2 fractures of the proximal part of the femur: a prospective randomized trial. J Bone Joint Surg Am. 2010;92:792-798.

5. Parker MJ, Handoll HH. Gamma and other cephalocondylic intramedullary nails versus extramedullary implants for extracapsular hip fractures in adults. Cochrane database Syst Rev. 2010:CD000093.

6. LCP Proximal Femoral Plate 4.5/5.0. Part of the LCP Periarticular Plating System. Oberdorf, Switzerland: Synthes GmbH. Available at: http://synthes.vo.llnwd.net/o16/LLNWMB8/INT\%20Mobile/Synthes\% 20International/Product\%20Support\%20Material/legacy_Synthes_PDF/ DSEM-TRM-0714-0121-2_LR.pdf.

7. Wieser K, Babst R. Fixation failure of the LCP proximal femoral plate 4.5/5.0 in patients with missing posteromedial support in unstable per-, inter-, and subtrochanteric fractures of the proximal femur. Arch Orthop Trauma Surg. 2010;130:1281-1287.

8. Glassner PJ, Tejwani NC. Failure of proximal femoral locking compression plate: a case series. J Orthop Trauma. 2011;25:76-83.

9. Streubel PN, Moustoukas MJ, Obremskey WT. Mechanical failure after locking plate fixation of unstable intertrochanteric femur fractures. J Orthop Trauma. 2013;27:22-28.

10. Wirtz C, Abbassi F, Evangelopoulos DS, et al. High failure rate of trochanteric fracture osteosynthesis with proximal femoral locking compression plate. Injury. 2013;44:751-756.

11. Collinge CA, Hymes R, Archdeacon M, et al. Unstable proximal femur fractures treated with proximal femoral locking plates: a retrospective, multicenter study of 111 cases. J Orthop Trauma. 2016;30:489-495.

12. Johnson B, Stevenson J, Chamma R, et al. Short-term follow-up of pertrochanteric fractures treated using the proximal femoral locking plate. J Orthop Trauma. 2014;28:283-287.

13. Schneider $\mathrm{K}$, Oh JK, Zderic I, et al. What is the underlying mechanism for the failure mode observed in the proximal femoral locking compression plate? A biomechanical study. Injury. 2015;46:1483-1490.

14. Collinge CA, Weber T, Watson T, et al. Results of Complex Proximal Femur Fractures Treated with Locking Proximal Femur Plates.
Proceedings of the 28 Annual OTA Meeting, October 3-6, 2012, Minneapolis, MN.

15. Marsh JL, Slongo TF, Agel J, et al. Fracture and dislocation classification compendium - 2007: orthopaedic Trauma Association classification, database and outcomes committee. J Orthop Trauma. 2007;21:S1-S133.

16. Bergmann G, Graichen F, Rohlmann A. Hip joint contact forces during stumbling. Langenbecks Arch Surg. 2004;389:53-59.

17. Lewis CL, Sahrmann SA, Moran DW. Effect of hip angle on anterior hip joint force during gait. Gait Posture. 2010;32:603-607.

18. Bergmann G, Deuretzbacher G, Heller M, et al. Hip contact forces and gait patterns from routine activities. J Biomech. 2001;34:859-871.

19. Gueorguiev B, Ockert B, Schwieger K, et al. Angular stability potentially permits fewer locking screws compared with conventional locking in intramedullary nailed distal tibia fractures: a biomechanical study. J Orthop Trauma. 2011;25:340-346.

20. Windolf M, Muths R, Braunstein V, et al. Quantification of cancellous bone-compaction due to DHS Blade insertion and influence upon cut-out resistance. Clin Biomech. 2009;24:53-58.

21. Connelly CL, Archdeacon MT. The lateral decubitus approach for complex proximal femur fractures: anatomic reduction and locking plate neutralization: a technical trick. J Orthop Trauma. 2012;26:252-257.

22. Cartner JL, Petteys T, Tornetta P, III. Mechanical effects of off-axis insertion of locking screws: should we do it? J Orthop Trauma. 2014;28 (suppl 1):S2-S5.

23. Kaab MJ, Frenk A, Schmeling A, et al. Locked internal fixator: sensitivity of screw/plate stability to the correct insertion angle of the screw. J Orthop Trauma. 2004;18:483-487.

24. Lenz M, Wahl D, Gueorguiev B, et al. Concept of variable angle locking - evolution and mechanical evaluation of a recent technology. J Orthop Res. 2015;33:988-992.

25. Lenz M, Wahl D, Zderic I, et al. Head-locking durability of fixed and variable angle locking screws under repetitive loading. J Orthopaedic Res. 2016;34:949-952.

26. Ehmke LW, Fitzpatrick DC, Krieg JC, et al. Lag screws for hip fracture fixation: evaluation of migration resistance under simulated walking. J Orthop Res. 2005;23:1329-1335.

27. Born CT, Karich B, Bauer C, et al. Hip screw migration testing: first results for hip screws and helical blades utilizing a new oscillating test method. J Orthop Res. 2011;29:760-766.

28. Santoni BG, Nayak AN, Cooper SA, et al. Comparison of femoral head rotation and varus collapse between a single lag screw and integrated dual screw intertrochanteric hip fracture fixation device using a cadaveric hemi-pelvis biomechanical model. J Orthop Trauma. 2016;30:164-169. 